\title{
Numerical relativity and the early Universe
}

\author{
Sergey Mironov ${ }^{1, \star}$ \\ ${ }^{1}$ Institute for Nuclear Research of the Russian Academy of Sciences, 60th October Anniversary Prospect, \\ 7a, 117312 Moscow, Russia
}

\begin{abstract}
We consider numerical simulations in general relativity in ADM formalism with cosmological ansatz for the metric. This ansatz is convenient for investigations of the Universe creation in laboratory with Galileons. Here we consider toy model for the software: spherically symmetric scalar field minimally coupled to the gravity with asymmetric double well potential. We studied the dependence of radius of critical bubble on the parameters of the theory. It demonstrates the wide applicability of thin-wall approximation. We did not find any kind of stable bubble solution.
\end{abstract}

\section{Introduction}

We study smooth solutions in spherically symmetric $3+1$ gravity with additional scalar field. This problem was studied in many aspects, both analytically [1-3] and numerically [4-6] (see [7] for a review). We return to this problem with Galileons in mind. There is an idea, that Galileon field allows one to create a new universe in the laboratory [8]. We just need to create special initial conditions to make some region in our nearly Minkowskian space-time undergo accelerated expansion and create a man-made universe. We want to simulate this process numerically. The most convenient way to do it is to use the following ansatz for the metric

$$
d s^{2}=\alpha^{2} d t^{2}+a^{2}\left(d r^{2}+r^{2} d \Omega^{2}\right) .
$$

We need to note, that the whole process is entirely smooth and always asymptotically Minkowskian. Initial configuration is just nearly a homogeneous field (of Galileon-type) in a sufficiently large sphere. The energy density is initially small everywhere, but increases inside the sphere, as the field evolves. While the expanding sphere becomes a new universe, the energy density outside remains small and asymptotes to zero at large distances.

But before proceeding to the numerical relativity with Galileons, we consider the toy model for software debugging: we examine the dynamics of bubbles of domain wall in the theory of standard scalar field minimally coupled to the gravity. The study of transitions in scalar fields from one minimum to another is itself very motivated by cosmology (mostly by early Universe inflationary-like models) and some classical field theory models.

The most popular topic in this context is a critical phenomena, first discovered by Choptuik [5]. Any family of initial configurations will evolve to one of the two end states - flat space (dispersal of

\footnotetext{
^e-mail: mironov@inr.ac.ru
} 
the field to infinity) or a black hole. Critical solution is a transition between these two end states. The non-trivial part is that in spherically symmetric collapse there is a peculiar relation between the mass $M$ of the formed black hole and any parameter $p$ of initial configuration family:

$$
M \sim\left(p-p_{*}\right)^{\gamma},
$$

here $p_{*}$ is a critical parameter and scaling index $\gamma$ does not depend on the choice and parametrization of initial family (only on the type of the matter). For a massless scalar field $\gamma \approx 0.37$ (which is a numerical result). The other interesting phenomenon is a self-similarity of the solution. It turns out that near the critical point field exhibits the following scaling relation,

$$
\phi(t, x)=\phi(\alpha t, \alpha x) \quad \alpha=e^{3.44} .
$$

It is seen in the simulations, that the same field profile appears on the $\alpha$ times smaller spatial scale, but $\alpha$ times closer to the critical time. Unfortunately, for now this result is not well understood and may be questionable.

But before moving to domain walls in general relativity let us, first, consider standard $\frac{\lambda}{4}\left(\varphi^{2}-v^{2}\right)^{2}$ potential without gravity. There is well-known classical solution: kink in $1+1$ dimensions, which generalizes to plain domain wall in $3+1$ dimensions

$$
\varphi=v \tanh \left(\sqrt{\frac{\lambda}{2}} v\left(x-x_{0}\right)\right) .
$$

It is a static solution, we can obtain moving one via boost. Spherically symmetric version of this solution is a bubble (in spherically symmetric situation analytic solution exists only in a thin-wall approximation). It could not be static due to the surface tension. So, we add small asymmetric term to the potential

$$
V(\varphi)=\frac{\lambda}{4}\left(\varphi^{2}-v^{2}\right)^{2}-\epsilon \varphi
$$

to obtain static solution (unstable). This solution is exactly a critical one.

The next step is to turn on gravity. First of all, in the thin-wall approximation [2]. In the next section we are going to describe the dynamic of bubbles in this case. In the third section, we will set up a full system of equations and consider numerical simulations. We conclude in the last section.

\section{Thin-wall approximation}

Thin-wall approximation in gravity-free case is quite trivial, since it is the limit of plain domain wall (4). Still it makes sense to review the dynamics of thin wall in the theory of scalar field with gravity. Let us start with the notations. We use Greek indices for four-dimensional tensors while Latin indices take three values and numerate components of the three-dimensional tensors of the hypersurface $(\Sigma)$. Superscripts + and - indicate outer and inner four-dimensional volumes from the different sides of the hypersurface. $\sigma_{m} u^{v}$ is a singular part of stress-energy tensor $T_{\mu}^{v}$ on the wall. $K_{i j}=-\Gamma_{i j}^{n}$ is outer curvature of $\Sigma$. Generally dynamic equations for the wall are the consequence of Einstein equations and junction conditions. First junction is a procedure of continuous matching of $g_{\mu \nu}^{-}$and $g_{\mu \nu}^{+}$on $\Sigma$. It allows one to write the metric on the whole manifold

$$
d s^{2}=\gamma_{i j} d x^{i} d x^{j}-d n^{2} .
$$


The Einstein equations ( $i, j$-components) with the use of $K_{i}^{j}$ and $\sigma_{i}^{j}$ could be presented in the following form

$$
\left(\delta_{i}^{j} K_{m}^{m}-K_{i}^{j}\right)^{+}-\left(\delta_{i}^{j} K_{m}^{m}-K_{i}^{j}\right)^{-}=8 \pi \kappa \sigma_{i}^{j} .
$$

In general Einstein equations and junction provide one with a lot of conditions (much more than functions to define) and not always possible to obey. The number of conditions is reduced due to additional symmetries, in our case - spherical symmetry.

$$
d s^{2}=g_{00} d t^{2}+2 g_{01} d t d q+g_{11} d q^{2}-r^{2} d \Omega^{2},
$$

as usual

$$
d \Omega^{2}=d \Theta^{2}+\sin ^{2}(\Theta) d \varphi^{2} .
$$

We require $g_{01}=0$ for simplicity (gauge freedom). The metric on the shell is

$$
d s^{2}=d \tau^{2}-\rho^{2}(\tau) d \Omega^{2} .
$$

The equations take the following form after imposing spherical symmetry:

$$
\begin{gathered}
4 \pi \kappa \sigma_{0}^{0}=-K_{2}^{2+}+K_{2}^{2-} \\
8 \pi \kappa \sigma_{2}^{2}=-K_{2}^{2+}+K_{2}^{2-}-K_{0}^{0+}+K_{0}^{0-}
\end{gathered}
$$

Since we are interested in bubbles of scalar field, we assume stress-energy tensor to be of special type, hence the first of the second junction conditions takes the form

$$
\left(1+\dot{\rho}^{2}-\frac{8 \pi \kappa}{3} \epsilon_{\text {in }} \rho^{2}\right)^{1 / 2}-\left(1+\dot{\rho}^{2}-\frac{8 \pi \kappa}{3} \epsilon_{\text {out }} \rho^{2}-\frac{2 \kappa m}{\rho}\right)^{1 / 2}=4 \pi \kappa \sigma_{0}^{0} \rho
$$

here $\epsilon_{\text {in }}$ is energy density in the inner volume. We will be interested in the case $\epsilon_{\text {out }}=0$, so we omit the subscript in. $\sigma$ here is a wall tension. Now it is possible to present this equation of motion for the bubble in two different forms

$$
\dot{\rho}^{2}=\frac{\left(\epsilon+6 \pi \kappa \sigma^{2}\right)^{2}}{9 \sigma^{2}} \rho^{2}-1+\frac{m}{\rho}\left(\kappa-\frac{\epsilon}{6 \pi S^{2}}\right)+\frac{m^{2}}{16 \pi^{2} S^{2} \rho^{4}}
$$

and

$$
m=\frac{4 \pi}{3} \epsilon \rho^{3}+4 \pi \rho^{2} S\left(\dot{\rho}^{2}+1-8 \pi \kappa \epsilon \rho^{2} / 3\right)^{1 / 2}-8 \pi^{2} \kappa S^{2} \rho^{3}
$$

It is easy to check, that there is no stable equilibrium point for domain wall. Any static solution is unstable (critical).

In other words, the effective potential has no minimum. Though, it is very close to having it, for example, an additional electric charge immediately stabilizes the bubble.

Now let us turn to the numerical simulations and check the presence of stable bubble there and applicability of thin-wall approximation to thick walls in general.

\section{Numerical setup}

We work in 3+1 ADM formalism [9], it is convenient for numerical simulations because equations are of the first order ${ }^{1}$. Equations of interest are projections of Einstein equations onto the hypersurface, on the normal and mixed projection [10]

$$
\left(\partial_{t}-\mathcal{L}_{\beta}\right) \gamma_{i j}=-2 \alpha K_{i j}
$$

\footnotetext{
${ }^{1}$ we use Planck units $\kappa=1$
} 


$$
\begin{gathered}
\left(\partial_{t}-\mathcal{L}_{\beta}\right) K_{i j}=-\nabla_{i} \nabla_{j} \alpha+ \\
+\alpha\left[R_{i j}+K K_{i j}-2 K_{i k} K_{j}^{k}+\frac{8 \pi}{D-2}(S-E) \gamma_{i j}-8 \pi S_{i j}\right] \\
R+K^{2}-K_{i j} K^{i j}=16 \pi E \\
\nabla_{j}\left(K^{i j}-\gamma^{i j} K\right)=8 \pi j^{i}
\end{gathered}
$$

For our purposes it is enough to consider spherical symmetry. In this case general expression for the metric is following

$$
d s^{2}=\left(-\alpha^{2}+a^{2} \beta^{2}\right) d t^{2}+2 a^{2} \beta d t d r+a^{2} d r^{2}+r^{2} b^{2} d \Omega^{2} .
$$

In standard framework $a$ and $b$ are dynamical variables and $\alpha$ and $\beta$ are gauge parameters. First of all, we impose an orthogonality condition $\beta=0$, this significantly simplifies all the equations. Among the dynamical variables there are also two extrinsic curvatures $K_{r}^{r}$ and $K_{\theta}^{\theta}$

$$
K_{i}^{j}=\operatorname{diag}\left(K_{r}^{r}, K_{\theta}^{\theta}, K_{\theta}^{\theta}\right)
$$

and scalar field variables $\Phi \equiv \phi^{\prime}$ and $\Pi \equiv \frac{a}{\alpha}\left(\dot{\phi}-\beta \phi^{\prime}\right)$. We consider real scalar field minimally coupled to the gravity.

$$
L=\frac{1}{2} \partial_{\mu} \phi \partial^{\mu} \phi+V(\phi)-\frac{1}{2} R
$$

The projected Einstein equations (16-19) and equations of motion for scalar field can be divided in two groups: dynamical equations (23-28) and constraints (29),(30).

We write them explicitly in the metric $d s^{2}=-\alpha^{2} d t^{2}+a^{2} d r^{2}+r^{2} b^{2} d \Omega^{2}$.

The dynamical equations for the metric components are

$$
\begin{aligned}
& \dot{a}=-a \alpha K_{r}^{r}, \\
& \dot{b}=-\alpha b K_{\theta}^{\theta} .
\end{aligned}
$$

The evolution equations for the extrinsic curvature components are

$$
\begin{gathered}
\dot{K}_{r}^{r}=\alpha K_{r}^{r}\left(K_{r}^{r}+2 K_{\theta}^{\theta}\right)-\frac{1}{a}\left(\frac{\alpha^{\prime}}{a}\right)^{\prime}-\frac{2 \alpha}{a r b}\left[\frac{(r b)^{\prime}}{a}\right]^{\prime}-8 \pi \alpha\left(\frac{\Phi^{2}}{a^{2}}+V(\phi)\right), \\
\dot{K}_{\theta}^{\theta}=\alpha K_{\theta}^{\theta}\left(K_{r}^{r}+2 K_{\theta}^{\theta}\right)+\frac{\alpha}{(r b)^{2}}-\frac{1}{a(r b)^{2}}\left(\frac{\alpha r b}{a}(r b)^{\prime}\right)^{\prime}-8 \pi \alpha V(\phi) .
\end{gathered}
$$

The dynamic of scalar field is determined by the following equations

$$
\begin{gathered}
\dot{\Phi}=\left(\frac{\alpha}{a} \Pi\right)^{\prime}, \\
\dot{\Pi}=\frac{1}{r^{2} b^{2}}\left[r^{2} b^{2}\left(\frac{\alpha}{a} \Phi\right)\right]^{\prime}-2 \Pi \frac{\dot{b}}{b}-\alpha a V_{\phi}(\phi),
\end{gathered}
$$




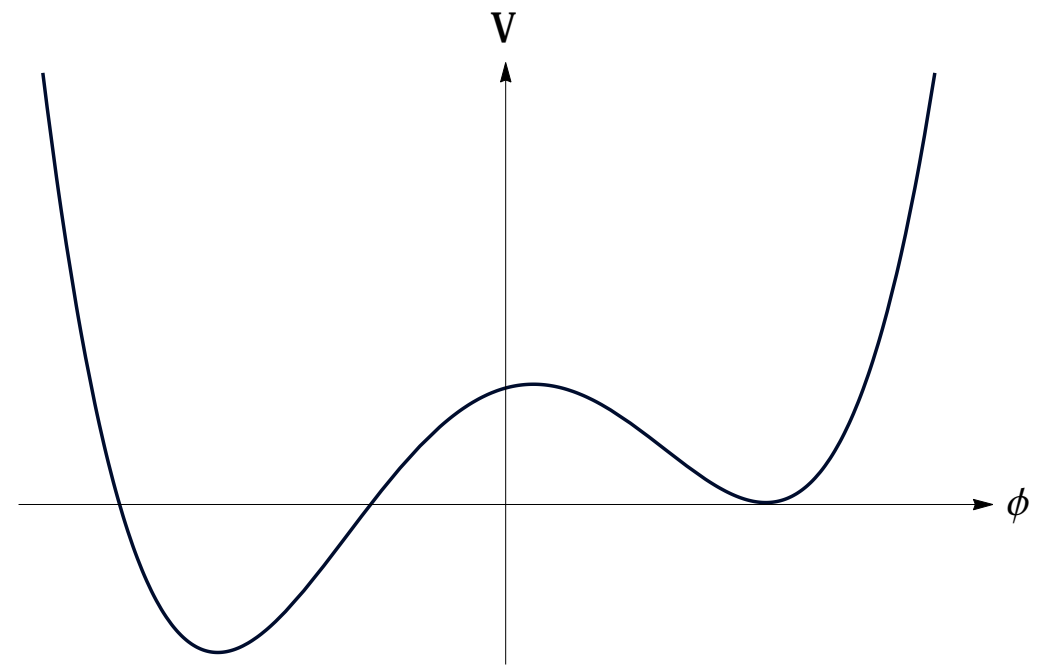

Figure 1. Double well asymmetric potential. We customize the value of the potential in the outside minimum to be zero.

In this ansatz the Hamiltonian constraint reads

$$
\begin{aligned}
-\frac{2}{a r b}\left[\left(\frac{(r b)^{\prime}}{a}\right)^{\prime}+\right. & \left.\frac{1}{r b}\left(\left(\frac{r b}{a}(r b)^{\prime}\right)^{\prime}-a\right)\right]+4 K_{r}^{r} K_{\theta}^{\theta}+2\left(K_{\theta}^{\theta}\right)^{2}= \\
& =8 \pi\left(\frac{\Phi^{2}+\Pi^{2}}{a^{2}}+2 V(\phi)\right)
\end{aligned}
$$

and the momentum constraint reads

$$
\frac{(r b)^{\prime}}{r b}\left(K_{\theta}^{\theta}-K_{r}^{r}\right)-\left(K_{\theta}^{\theta}\right)^{\prime}=-4 \pi \frac{\Phi \Pi}{a}
$$

We consider bubbles of domain wall interpolating between two minima. We customize the value of the potential in the outside minimum to be zero, hence on the spatial infinity metric is Schwarzschild without cosmological constant. Inside the bubble cosmological constant is negative.

$$
V(\phi)=\frac{\lambda}{4}\left(\phi^{2}-v^{2}\right)^{2}+\varepsilon(\phi-v)+c
$$

Now, instead of fixing $\alpha$, we equate $a$ and $b$ while keeping $\alpha$ free ${ }^{2}$. This is a convenient ansatz for our later objectives with Galileons and Universe creation. In other words we customize the gauge

${ }^{2}$ It worth noting that Schwarzschild metric in our ansatz is a bit different:

$$
\alpha=\sqrt{1-\frac{2}{\frac{r}{m}+\frac{m}{4 r}+1}} \quad a=1+\frac{m}{r}+\frac{m^{2}}{4 r^{2}}
$$


condition so that the dynamical variables $a$ and $b$ are equal. This condition combined with (23),(24) provides $K_{r}^{r}=K_{\theta}^{\theta} \equiv K$. After this procedure we are left with four dynamical equations: one for metric (now (23) and (24) are the same), one for curvature (either (25) or (26)) and still two for scalar field. In addition we got a constraint equation to determine $\alpha$, it comes from the difference of (25) and (26).

We apply these five equations to evaluate initial configuration, but because of unstable mode we are also forced to keep the constrains (29),(30) satisfied. As for initial conditions, we specify scalar field as domain wall of given radius and thickness and solve the constraint equations to determine $\alpha$, $a$ and $K$.

\section{Conclusions}

We designed and debugged a software for numerical simulations in gravity with scalar fields. The main difference from previous works is the special ansatz for the metric and hence slightly different concept with initial conditions and equations of motion. We studied how gravity affects the dynamics of bubbles in the scalar theory in our ansatz but did not find any kind of stable bubble solution. We see that the thin-wall approximation is applicable for a wide range of bubble configurations, even with fairly thick walls.

Subsequently we plan to gather more data and examine the detailed dependence of the radius of critical bubble on the parameters of the model. Moreover, we naturally are going to simulate the universe creation with Galileon field.

Authors are indebted to Valery Rubakov for useful discussions and inspiration. Authors want to thank Diego Blas and Victoria Volkova for comments and interest to the work. This work was supported by Russian Science Foundation grant 14-22-00161

\section{References}

[1] V. Berezin, V. Kuzmin, I. Tkachev, Phys.Lett. B120 (1983) 91-96

[2] V. Berezin, V. Kuzmin, I. Tkachev, Phys.Rev. D36 (1987) 2919

[3] Y. Takamizu, K. Maeda, Phys.Rev. D92 (2015) no.2, 023514, arXiv:1504.05788

[4] R. Marsa, M. Choptuik, Phys.Rev. D54 (1996) 4929-4943, arXiv:gr-qc/9607034

[5] M. Choptuik, Phys.Rev.Lett. 70 (1993) 9-12

[6] K. Clough, E. Lim, arXiv:1602.02568

K. Clough, P. Figueras, H. Finkel, M. Kunesch, E. Lim, S. Tunyasuvunakool, Class.Quant.Grav. 32 (2015) no.24, 245011, Class.Quant.Grav. 32 (2015) 24, arXiv:1503.03436

[7] C. Gundlach, J. Martin-Garcia, Living Rev.Rel. 10 (2007) 5, arXiv:0711.4620

[8] V. Rubakov, Phys.Rev. D88 (2013) 044015, arXiv:1305.2614

[9] R. Arnowitt, S. Deser, C. Misner, Gen.Rel.Grav. 40 (2008) 1997-2027,arXiv: gr-qc/0405109

R. Arnowitt, S. Deser, C. Misner, Phys.Rev. 117 (1960) 1595-1602

[10] M. Zilhao, arXiv:1301.1509 